\title{
Exact Bound State Solutions of the Schrödinger Equation for Noncentral Potential via the Nikiforov-Uvarov Method
}

\author{
Metin Aktaş* \\ Department of Physics, Faculty of Arts and Sciences \\ Kırıkkale University, 71450, Kırıkkale, Turkey
}

October 3, 2018

\begin{abstract}
Exact bound state solutions of the Schrödinger equation for generalized noncentral potential are examined by means of the Nikiforov-Uvarov method. The wavefunctions and the corresponding energy eigenvalues of the system are obtained analytically. The results examined for the potential are compatible with those obtained by the other methods.
\end{abstract}

Keywords: Schrödinger Equation, Noncentral Potential, Nikiforov-Uvarov method.

PACS numbers: 02.30.Hq, 03.65.Ca, 03.65.Fd, 03.65.Ge, 04.20.Jb

\footnotetext{
${ }^{*}$ Corresponding author: E-mail: metin@kku.edu.tr
} 


\section{Introduction}

Exact analytic studies on the Schrödinger equation for noncentral potentials have been considerable interests in recent years. Some of the numerous studies concerning in the context are available in the literature [1-7]. Two different classes of the potentials are considered in searching both relativistic and nonrelativistic quantum mechanical systems. The first case is Coulombic type potential which is used in analyzing the bound states of an electron within the Hartmann's ring-shaped plus Aharonov-Bohm (AB) field [8] or a magnetic Dirac monopole [9]. They can be used in quantum chemistry for describing the ring-shaped organic molecules such as cyclic polyenes and benzene as well as in nuclear physics for investigating the interaction between deformed pair of nuclei and the spinorbit coupling for the motion of the particle in the potential fields. The second one is the generalized harmonic oscillator type potential known also as the ring-shaped oscillator plus AB-systems [10]. These are very useful for calculating the vibrational quantum levels and determining the hidden dynamical symmetries of quantum systems which are described a particle moving in the potential fields. In this study, we introduce a ring-shaped type generalized noncentral potential and investigate its solutions with respect to the Schrödinger equation.

By replacing the Coulomb term of the Hartmann's potential with the harmonic oscillator one and adding to the inverse square term (or saying that it is either attractive or repulsive for negative and positive parameters) respectively. It also includes contribution of the potential term as $(r \cos \theta)^{-2}$. Thus, we define the potential in spherical coordinates as follows:

$$
V_{q}(r, \theta)=\left(A r^{2}+\frac{B}{r^{2}}\right)+\frac{q}{r^{2}}\left(\frac{C}{\sin ^{2} \theta}+\frac{D}{\cos ^{2} \theta}\right)
$$

where $q$ is the deformation parameter [11]. In equation (1), only $\theta$-dependent part $V(\theta)=$ $\left(\frac{C}{\sin ^{2} \theta}+\frac{D}{\cos ^{2} \theta}\right)$ is often called the Pöschl-Teller I type potential with $C=\frac{V_{0}}{2} \chi(\chi-1)$,

$D=\frac{V_{0}}{2} \lambda(\lambda-1)$ and $V_{0}=\frac{\hbar^{2} \alpha^{2}}{m}[12]$. Various choices of energy parameters allow us to investigate for some particular cases:

For $B=0$, the potential (1) turns into the double ring-shaped oscillator [13]; for $B=D=0$, the ring-shaped oscillator $[11,13]$. Moreover, for only $D=0$, it refers to the nonspherical ring-shaped oscillator [2]; for only $q=0$ or $C=D=0$, the harmonic oscillator plus inverse square potentials, respectively. Besides these, for $B=C=D=0$, the usual spherical harmonic oscillator potential is possible.

Noncentral potentials are not usually discussed in quantum mechanical problems. The main reason is that most of them are not analytically solvable. In spite of this fact, some classes of noncentral potentials in three dimensions are solvable so long as the Schrödinger, Klein-Gordon and Dirac equations [14-26] with these potentials satisfy the separation of variables. These are also applicable to the scattering and condensed matter processes 
[27-30]. There have been many studies involving the potentials by using the well-known techniques, i.e., group theoretical manner [11, 31-33], supersymmetric formalism [34-41], path integral method [42-48] and other algebraic approaches [49-58].

Throughout the study, we have focused on several purposes. One of them is to solve the separated Schrödinger equations for the potential (1) by using the Nikiforov-Uvarov (NU) method [62] analytically. Our another interest is to extract some remarkable results.

The outline of the paper is as follows: In Sec. II, the method is introduced briefly. In Sec. III, the wavefunction and the corresponding energy eigenvalues are obtained analytically. In Sec. IV, our results are reviewed and discussed.

\section{The Method: Description of the Procedure}

Let us first introduce the Nikiforov-Uvarov method. Some quantum mechanical problems can be solved analytically after applying the separation of variables procedure [7, 49]. Then, a proper transformation is chosen as $x=x(z)$ for each separated equation. Therefore, each of them is reduced to a generalized equation of hypergeometric type as [62]

$$
u^{\prime \prime}(z)+\frac{\tilde{\tau}(z)}{\sigma(z)} u^{\prime}(z)+\frac{\tilde{\sigma}(z)}{\sigma^{2}(z)} u(z)=0 .
$$

Here, $\tilde{\tau}(z)$ is a polynomial function with the highest degree 1 and $\sigma(z)$ and $\tilde{\sigma}(z)$ are polynomial functions with the highest degree 2 . It can also be noted that the energy $E_{n}$ appear as a parameter in these coefficients. Their solutions, therefore, lead to several classes of special functions such the classical orthogonal polynomials called as Hermite, Jacobi, Bessel etc. If we apply the transformation $u(z)=\phi(z) y(z)$ to original equation (3) and arrange it, we have a simpler form as

$$
\sigma(z) y^{\prime \prime}+\tau(z) y^{\prime}+\lambda y=0
$$

Here, one concludes

$$
\frac{\pi(z)}{\sigma(z)}=\frac{d}{d z}[\ln \phi(z)]
$$

and

$$
\tau(z)=\tilde{\tau}(z)+2 \pi(z)
$$

Moreover, the nontrivial solutions of equation (4) must satisfy the energy eigenvalue equation

$$
\lambda_{n}+n \tau^{\prime}+\frac{1}{2}\left[n(n-1) \sigma^{\prime \prime}\right]=0, \quad(n=0,1,2 \ldots)
$$


where $\tau^{\prime}$ and $\sigma^{\prime \prime}$ denote the first and second derivatives of them with respect to $z$. To determine $\pi(z)$ and $\lambda=\lambda_{n}$ by assuming

$$
k=\lambda-\pi^{\prime}(z)
$$

and by solving the quadratic equation for $\pi(z)$, one gets

$$
\pi(z)=\left(\frac{\sigma^{\prime}-\tilde{\tau}}{2}\right) \pm \sqrt{\left(\frac{\sigma^{\prime}-\tilde{\tau}}{2}\right)^{2}+k \sigma-\tilde{\sigma}}
$$

with the prime factors of $\sigma$ denoted as the differential at first degree. The polynomial expression $\pi(z)$ has to be the square of polynomials under the square root sign. The existence is possible only if its discriminant is zero. Thus, it takes the form without square root. We also point out that the determination of $k$ is the essential point in the calculation of $\pi(z)$. Our problem with a nontrivial solutions corresponds to the eigenfunctions $y_{n}(z)$ called as the Rodriguez formula

$$
y_{n}(z)=\frac{C_{n}}{\rho(z)} \frac{d^{n}}{d z^{n}}\left[\sigma^{n}(z) \rho(z)\right]
$$

where $C_{n}$ and $\rho(z)$ are the normalization constants and the weight function, respectively. This function must satisfy the condition

$$
\rho(z) \tau(z)=\frac{d}{d z}[\sigma(z) \rho(z)]
$$

Therefore, wavefunctions corresponding to discrete eigenvalues are orthonormal that they comply with

$$
\int y_{n}(z) y_{m}(z) \rho(z) d z=\delta_{m n}
$$

If we further specify that the wavefunctions can be normalized when $m=n$.

\section{Calculations}

For a particle in any potential field, the wave functions $\Psi_{n}(\mathbf{r})$ describe the bound states and the corresponding energy levels $E_{n}$, then the time-independent Schrödinger wave equation becomes

$$
\nabla^{2} \Psi(\mathbf{r})+\frac{2 m}{\hbar^{2}}[E-V(\mathbf{r})] \Psi(\mathbf{r})=0
$$


Let us first consider this equation. By putting the potential $V(\mathbf{r})=V_{q}(r, \theta)$ into the equation in spherical coordinates, one has

$$
\begin{aligned}
\frac{1}{r^{2}} \frac{\partial}{\partial r}\left(r^{2} \frac{\partial \Psi_{E}}{\partial r}\right) & +\frac{1}{r^{2}}\left[\frac{1}{\sin \theta} \frac{\partial}{\partial \theta}\left(\sin \theta \frac{\partial \Psi_{E}}{\partial \theta}\right)+\frac{1}{\sin ^{2} \theta} \frac{\partial^{2} \Psi_{E}}{\partial \varphi^{2}}\right] \\
& +\frac{2 m}{\hbar^{2}}\left\{E-\left[\left(A r^{2}+\frac{B}{r^{2}}\right)+\frac{q}{r^{2}}\left(\frac{C}{\sin ^{2} \theta}+\frac{D}{\cos ^{2} \theta}\right)\right]\right\} \Psi_{E}=0 .
\end{aligned}
$$

By taking the total wave function as

$$
\begin{aligned}
\Psi(\mathbf{r}) & =\Psi_{E}(r, \theta, \varphi) \\
& =\frac{1}{r} R(r) \Theta(\theta) \Phi(\varphi),
\end{aligned}
$$

and substituting it into equation (13), one brings to the forms

$$
\frac{d^{2} R}{d r^{2}}+\left[\varepsilon-\left(\bar{A} r^{2}+\frac{\gamma}{r^{2}}\right)\right] R(r)=0
$$

and

$$
\frac{d^{2} \Theta}{d \theta^{2}}+\cot \theta \frac{d \Theta}{d \theta}+\left[\Lambda-\left(\frac{\widetilde{C}}{\sin ^{2} \theta}+\frac{\bar{D}}{\cos ^{2} \theta}\right)\right] \Theta=0
$$

with the well-known $\varphi$-angle (or azimuthal) solution

$$
\Phi_{\bar{m}}(\varphi)=\frac{1}{\sqrt{2 \pi}} e^{i \bar{m} \varphi}, \quad \bar{m}=0, \pm 1, \pm 2 \ldots
$$

Equations (15) and (16) are called radial and $\Theta$-angle equations, respectively and they are in separated forms. In equations (15) and (16), $\varepsilon=\varepsilon(A, B, \gamma)=\frac{2 m E}{\hbar^{2}}, \bar{A}=\frac{2 m A}{\hbar^{2}}$, $\bar{B}=\frac{2 m B}{\hbar^{2}}, \bar{C}=\frac{2 q m C}{\hbar^{2}}$ and $\bar{D}=\frac{2 q m D}{\hbar^{2}}$ and also $\widetilde{C}=\bar{C}+\bar{m}^{2}, \gamma=\bar{B}+\Lambda$ are defined. Here, $\bar{m}^{2}$, and $\Lambda=\Lambda(C, D, \bar{m})$ are known as separation constants. By using the NU-method, we seek for the solutions of the equations.

\subsection{Energy Eigenvalues}

\subsubsection{Radial equation}

Let us now consider equation (15) only if $\varepsilon<0$. When we apply a transformation $z=r^{2}$ to it, then we get the transformed hypergeometric equation 


$$
R^{\prime \prime}(z)+\frac{1}{2 z} R^{\prime}(z)+\frac{1}{4 z^{2}}\left[-\bar{A} z^{2}-\varepsilon z-\gamma\right] R(z)=0 .
$$

By comparing it with equation (2), one has

$$
\tilde{\tau}(z)=1, \quad \sigma(z)=2 z, \quad \tilde{\sigma}(z)=\left[-\bar{A} z^{2}-\varepsilon z-\gamma\right] .
$$

When we substitute these polynomials into equation (8), we obtain

$$
\pi(z)=\frac{1}{2} \pm \frac{1}{2} \sqrt{4 \bar{A} z^{2}+4(\varepsilon+2 k) z+(1+4 \gamma)} .
$$

From this equation, $k$ can be determined and we rewrite it as

$$
\pi(z)=\frac{1}{2} \pm \frac{1}{2} \begin{cases}(2 \sqrt{\bar{A}} z-\sqrt{1+4 \gamma}), & k=-\frac{\varepsilon}{2}-\frac{1}{2} \sqrt{\bar{A}(1+4 \gamma)} \\ (2 \sqrt{\bar{A}} z+\sqrt{1+4 \gamma}), & k=-\frac{\varepsilon}{2}+\frac{1}{2} \sqrt{\bar{A}(1+4 \gamma)}\end{cases}
$$

So the proper are the values of $\pi(z)$ that they must satisfy the condition $\tau^{\prime}(z)<0$ and we have two cases:

(i) $\pi=\pi_{1}(z)=\frac{1}{2}-\frac{1}{2}(2 \sqrt{\bar{A}} z-\sqrt{1+4 \gamma}) \quad$ for $\quad k=k_{1}=-\frac{\varepsilon}{2}-\frac{1}{2} \sqrt{\bar{A}(1+4 \gamma)}$

In this time we get

$$
\begin{aligned}
\lambda=\lambda_{n} & =-\frac{\varepsilon}{2}-\frac{1}{2} \sqrt{\bar{A}(1+4 \gamma)}-\sqrt{\bar{A}} \\
& =2 n \sqrt{\bar{A}} .
\end{aligned}
$$

As a result, it gives us the energy eigenvalue equation

$$
E_{n}=\widetilde{A}\left[(2 n+1)+\frac{1}{2} \sqrt{1+4 \gamma}\right], \quad n=0,1,2 \ldots
$$

where $\widetilde{A}=\frac{\hbar^{2}}{m} \sqrt{\bar{A}}$.

(ii) $\pi=\pi_{2}=\frac{1}{2}-\frac{1}{2}(2 \sqrt{\bar{A}} z+\sqrt{1+4 \gamma}) \quad$ for $\quad k=k_{2}=-\frac{\varepsilon}{2}+\frac{1}{2} \sqrt{\bar{A}(1+4 \gamma)}$

By recalculating $\lambda=\lambda_{n}$ and arranging it, one gets

$$
E_{n}=\tilde{A}\left[(2 n+1)-\frac{1}{2} \sqrt{1+4 \gamma}\right], \quad n=0,1,2 \ldots
$$

We note that these two eigenvalue solutions are obtained by following the conditions in equation (21). The first one refers to the solutions of 3 -dimensional harmonic oscillator, by taking care of the parameters given below of equation (17) as $B=0$ and $\gamma \equiv \Lambda=\ell(\ell+1)$. 


\subsection{2 $\Theta$-Angle Equation}

In order to solve equation (16) by the NU-method, we need to recast it into a new solvable form. Thus we introduce

$$
y^{\prime \prime}(x)+f(x) y^{\prime}(x)+g(x) y(x)=0,
$$

with $y(x)=v(x) p(x)$. Then, it is transformed to

$$
v^{\prime \prime}(x)+\left(2 \frac{p^{\prime}}{p}+f\right) v^{\prime}(x)+\frac{1}{p}\left(p^{\prime \prime}+f p^{\prime}+g p\right) v(x)=0,
$$

where we have $p(x)=\exp \left[-\frac{1}{2} \int f(x) d x\right]$. By applying the above procedure for equation (16) and taking $v \rightarrow \Theta$, one yields

$$
\Theta^{\prime \prime}(\theta)+\left[\Gamma-\left(\kappa \operatorname{cosec}^{2} \theta+\bar{D} \sec ^{2} \theta\right)\right] \Theta(\theta)=0
$$

where $\quad \Gamma=(L+1 / 2)^{2}=(\Lambda+1 / 4), L$ is used instead of $\ell$, and $\kappa=(\widetilde{C}-1 / 4)$. By introducing a new variable as $\sqrt{t}=\sin \theta$, equation (27) now reads

$$
\Theta^{\prime \prime}(t)+\frac{\left(\frac{1}{2}-t\right)}{t(1-t)} \Theta^{\prime}(t)+\frac{1}{[t(1-t)]^{2}}[\widetilde{\Gamma} t(1-t)-\widetilde{\kappa}(1-t)-\widetilde{D} t] \Theta(t)=0
$$

Here, we have $\widetilde{\Gamma}=\Gamma / 2, \widetilde{\kappa}=\kappa / 4$ and $\widetilde{D}=\bar{D} / 4$. Again comparing it with equation (2), one has

$$
\tilde{\tau}(t)=\left(\frac{1}{2}-t\right), \quad \sigma(t)=t(1-t), \quad \tilde{\sigma}(t)=\left[-\widetilde{\Gamma} t^{2}+\zeta t-\widetilde{\kappa}\right]
$$

with $\zeta=(\widetilde{\Gamma}+\widetilde{\kappa}-\widetilde{D})$. By substituting these polynomials into equation (8), one gets

$$
\pi(t)=\frac{1}{4}(1-2 t) \pm \frac{1}{4} \sqrt{a_{1} t^{2}+b_{1} t+c_{1}}
$$

Here, $a_{1}, b_{1}$ and $c_{1}$ parameters are equal to $(4+16 \widetilde{\Gamma}-16 \widetilde{\kappa}),(4-16 \zeta+16 k)$ and $(1+16 \widetilde{\kappa})$, respectively. From this equation, $k$ is determined and we rewrite it 


$$
\begin{aligned}
& \pi(t)=\frac{1}{4}(1-2 t) \pm \frac{1}{4} \\
& \left\{\begin{array}{l}
{[\sqrt{(1+4 \kappa)}+\sqrt{(1+4 \bar{D})}] t-\sqrt{(1+4 \kappa)}, \quad k=\frac{1}{8}+\frac{1}{4}[\Gamma-(\kappa+\bar{D})]-\frac{1}{8} \sqrt{(1+4 \kappa)(1+4 \bar{D})}} \\
{[\sqrt{(1+4 \kappa)}-\sqrt{(1+4 \bar{D})}] t-\sqrt{(1+4 \kappa)}, \quad k=\frac{1}{8}+\frac{1}{4}[\Gamma-(\kappa+\bar{D})]+\frac{1}{8} \sqrt{(1+4 \kappa)(1+4 \bar{D})} .}
\end{array}\right.
\end{aligned}
$$

Proper values of $\pi(t)$ can be chosen so that they must satisfy $\tau^{\prime}(t)<0$. Two possible cases are valid as follows:

(i) Having $\pi=\pi_{1}(t)=\frac{1}{4}(1-2 t)-\frac{1}{4}\{[\sqrt{(1+4 \kappa)}+\sqrt{(1+4 \bar{D})}] t-\sqrt{(1+4 \kappa)}\}$ for $k=k_{3}=\frac{1}{8}+\frac{1}{4}[\Gamma-(\kappa+\bar{D})]-\frac{1}{8} \sqrt{(1+4 \kappa)(1+4 \bar{D})}$, we get

$$
\begin{aligned}
\lambda=\lambda_{\bar{n}}= & \bar{n}^{2}+\bar{n}\left\{1+\frac{1}{2}[\sqrt{(1+4 \kappa)}+\sqrt{(1+4 \bar{D})}]\right\} \\
= & -\frac{3}{8}+\frac{1}{4}[\Gamma-(\kappa+\bar{D})]-\frac{1}{8} \sqrt{(1+4 \kappa)(1+4 \bar{D})} \\
& -\frac{1}{4}[\sqrt{(1+4 \kappa)}+\sqrt{(1+4 \bar{D})}]
\end{aligned}
$$

By putting the potential parameters cautiously into the equation and rearranging its right hand sides only, one obtains

$$
\begin{aligned}
\Lambda & \equiv L(L+1) \\
& =\left(s_{1}-\frac{1}{2}\right)\left(s_{1}+\frac{1}{2}\right),
\end{aligned}
$$

with the compact parameter

$s_{1}=\sqrt{(2 \bar{n}+1)[(2 \bar{n}+1)+(\sqrt{(1+4 \kappa)}+\sqrt{(1+4 \bar{D})})]+\frac{1}{2}[1+\sqrt{(1+4 \kappa)(1+4 \bar{D})}]+(\kappa+\bar{D})}$.

(ii) The other solution is

$$
\begin{aligned}
& \pi=\pi_{2}(t)=\frac{1}{4}(1-2 t)-\frac{1}{4}\{[\sqrt{(1+4 \kappa)}-\sqrt{(1+4 \bar{D})}] t-\sqrt{(1+4 \kappa)}\} \\
& \text { for } k=k_{4}=\frac{1}{8}+\frac{1}{4}[\Gamma-(\kappa+\bar{D})]+\frac{1}{8} \sqrt{(1+4 \kappa)(1+4 \bar{D})}, \text { then we have }
\end{aligned}
$$




$$
\begin{aligned}
\Lambda & \equiv L(L+1) \\
& =\left(s_{2}-\frac{1}{2}\right)\left(s_{2}+\frac{1}{2}\right),
\end{aligned}
$$

with the compact parameter

$$
s_{2}=\sqrt{(2 \bar{n}+1)^{2}-(2 \bar{n}+1)[\sqrt{(1+4 \kappa)}-\sqrt{(1+4 \bar{D})}]+\frac{1}{2}[1-\sqrt{(1+4 \kappa)(1+4 \bar{D})}]+(\kappa+\bar{D})} .
$$

Within the framework of NU-method, the procedure which solutions are considered physically acceptable is important. As the last two equations satisfy the rule of $\tau^{\prime}(t)<0$ [62], both are acceptable solutions.

\subsection{Wavefunctions}

\subsubsection{Radial wavefunction}

To determine the wavefunctions, we first consider equation (4) and we get

$$
\phi(z)=z^{\delta / 4} e^{-\frac{1}{2} \sqrt{A} z}
$$

where $\delta=(1+\sqrt{1+4 \gamma})$ and $\sqrt{z}=r$. From equation (10) by calculating $\rho(z)$ and inserting it into equation (9), it stands for the generalized Laguerre polynomials as

$$
y_{n}(z)=\tilde{C}_{n} L_{n}^{\mu}(z)
$$

with $\mu=(\delta-1) / 2$ and the weight function is $\rho(z)=z^{(\delta-1)} e^{-\sqrt{A} z}$. Hence, the radial wave functions become

$$
\begin{aligned}
R(z) & =\phi(z) y_{n}(z) \\
& =\tilde{C}_{n} z^{\delta / 4} e^{-\frac{1}{2} \sqrt{A} z} L_{n}^{\mu}(z),
\end{aligned}
$$

where $\tilde{C}_{n}$ are normalization constants. When we insert the weight function $\rho(z)$ and equation (36) in equation (11), we obtain

$$
\left|\tilde{C}_{n}\right|=\left(\frac{(-1)^{-n}(n !)(\bar{A})^{(\delta-1) / 4}}{\sqrt{(n+\delta-1) !}}\right), \quad n=0,1,2, \ldots
$$




\subsection{2 $\Theta$-Angle Wavefunction}

From equation (4), one finds

$$
\phi(t)=[t(1-t)]^{\left(1+\frac{\Delta}{2}\right) / 2},
$$

with $\Delta=[\sqrt{(1+4 \kappa)}+\sqrt{(1+4 \bar{D})}]$. By obtaining $\rho(t)$ via equation (10) and substituting it into equation (9), it stands for the Jacobi polynomials as

$$
y_{\bar{n}}(t)=\tilde{C}_{\bar{n}} P_{\bar{n}}^{\left(\nu_{1}, \nu_{2}\right)}(t)
$$

where $\nu_{1}=\sqrt{(1+4 \kappa)}, \nu_{2}=\sqrt{(1+4 \bar{D})}$ and $\tilde{C}_{\bar{n}}$ are normalization constants. Also, $\rho(t)=$ $[t(1-t)]^{\Delta / 2}$ is used. Thus we can write

$$
\begin{aligned}
\Theta(t) & =\phi(t) y_{\bar{n}}(t) \\
& =\tilde{C}_{\bar{n}}[t(1-t)]^{\left(1+\frac{\Delta}{2}\right) / 2} P_{\bar{n}}^{\left(\nu_{1}, \nu_{2}\right)}(t),
\end{aligned}
$$

with $\sqrt{t}=\sin \theta$. By means of the similar procedure as in radial part, we get

$$
\left|\tilde{C}_{\bar{n}}\right|=\frac{(2 \bar{n}+\Delta) !}{\left(\bar{n}+\frac{\Delta}{2}\right) !} \sqrt{\frac{(2 \bar{n}+\Delta+1)}{(\bar{n} !)(\Delta !)}}, \quad \bar{n}=0,1,2, \ldots
$$

\subsubsection{Total Wavefunctions}

By following equation (14) and gathering equations (17), (37), (40) respectively, one can construct the total wave function in compact form

$$
\Psi(z, t, \varphi)=\widetilde{C}_{n, \bar{n}} z^{(\delta-1) / 4} e^{-\frac{1}{2} \sqrt{A} z} L_{n}^{\mu}(z)[t(1-t)]^{\left(1+\frac{\Delta}{2}\right) / 2} P_{\bar{n}}^{\left(\nu_{1}, \nu_{2}\right)}(t) e^{i \bar{m} \varphi},
$$

or it can also be written as

$$
\Psi(r, \theta, \varphi)=\widetilde{C}_{n, \bar{n}} r^{(\delta-1) / 2} e^{-\frac{1}{2} \sqrt{A} r^{2}} L_{n}^{\mu}\left(r^{2}\right) \quad(\sin \theta \cos \theta)^{\left(1+\frac{\Delta}{2}\right)} P_{\bar{n}}^{\left(\nu_{1}, \nu_{2}\right)}\left(\sin ^{2} \theta\right) e^{i \bar{m} \varphi},
$$

with $\widetilde{C}_{n, \bar{n}}=\frac{\left|\tilde{C}_{n}\right|\left|\tilde{C}_{\bar{n}}\right|}{\sqrt{2 \pi}}$. 


\section{Concluding Remarks}

In this paper, bound-state solutions of the Schrödinger equation for $(r, \theta)$ dependent generalized noncentral potential have been investigated by the Nikiforov-Uvarov method. Both the wavefunctions and the corresponding energy spectra of the system have an exact and explicit forms. Some remarkable results are noted. By various choices of potential parameters, our results can also be reduced to the solutions of some quantum mechanical systems. Possible cases and results are as follows:

(i) For example, equation (23) turns into the energy eigenvalues of the spherical harmonic oscillator as

$$
E_{n}=\hbar \omega\left(2 n_{r}+\ell+\frac{3}{2}\right)
$$

for cases $A=\frac{m \omega^{2}}{2}, B=C=D=0$ and $\gamma=\Lambda=\ell(\ell+1)$, where $2 n_{r}+\ell=n$ (i.e., called as the principal quantum number). It is noted that the energy levels of the system are degenerate except for the ground state $n=0$. The degeneracy of the system is increased by the larger values of the radial quantum number $n_{r}$ as well as the orbital angular momentum quantum number $\ell$.

(ii) The deformation parameter $q$, in equation (1), takes some values as [11]. If we take $q=0$ or $C=D=0$ in equation (1), we can thus get the solutions of harmonic oscillator plus inverse square potential. To run the procedure, we should follow the equations from (13) to (16) and consider the parameters in Section III as well as the equation (23), our result becomes

$$
E_{n}=\widetilde{A}\left[(2 n+1)+\sqrt{\left(\ell+\frac{1}{2}\right)^{2}+\frac{2 m B}{\hbar^{2}}}\right] .
$$

(iii) For $q=1$, our results of the ring-shaped oscillator potential for $(B=D=0)$ and that of axially symmetric potential for $B=0$ are identical to those of the systems in $[11$, 13], respectively.

$(i v)$ If all the energy parameters are chosen greater than zero, the eigenvalue equation is therefore written with equation (33) or equation (34)

$$
E_{n}=\widetilde{A}\left[(2 n+1)+\sqrt{1+4 \Lambda+\frac{2 m B}{\hbar^{2}}}\right] .
$$

This is the most general energy eigenvalue result of the system.

$(v)$ When $A=0$, in equation (1), the bound state equation (23) vanishes or collapses to zero. It implies the second type bound state (called as pseudo-bound state) for nonlocal 
interactions in three-body or N-body systems [59-61]. For $A \rightarrow 0$, the energy spectrum (23) may however approach to a continuum bound state (BCS) depending on the coupling parameters $B, C, D$ and the azimuthal quantum number $(\bar{m})$. The occurrence of a BCS is hence only a necessary but not a sufficient condition for such an unphysical collapse in few body systems.

(vi) Equation (15) at $r=0$; equations (16), (27) at $\theta=0$ and $\theta=\pi / 2$; and equation (28) at $t=0$ and $t=1$ have singularities. It implies that their solutions are analytic for $t \epsilon[0,1]$, and either $\theta \epsilon[0, \pi / 2]$ or $\theta \epsilon[\pi / 2, \pi]$ intervals. Also, the wavefunctions (41) and (42) vanish at points $z=0, t=0$ and $t=1 ; r=0$ and $\theta=0, \pi / 2$, respectively. As a result, radial and angular components $(r, \theta)$ and transformation parameters $(z, t)$ in wavefunctions should not take these values.

(vii) By introducing the appropriate transformation, we get the solvable form of the $\Theta-$ angle equation (16) which is transformed to the equation (27) and (28). Then, $\theta$-dependent part solutions of (27) refer to bound-state solutions of the Pöschl-Teller potential I by putting $\Gamma \rightarrow E, \kappa \rightarrow \frac{V_{0}}{2} \chi(\chi-1), \bar{D} \rightarrow \lambda(\lambda-1)$ and by mapping $\theta \rightarrow x$ in [12]. 


\section{Acknowledgment}

This work is supported by the Turkish Scientific and Technological Research Council (TUBITAK). 


\section{References}

[1] Dong, S. H., Sun, G. H., Cassou, M. L.: Phys. Lett. A 340, 94 (2005); 328, 299 (2004)

[2] Chen C. Y., Dong, S. H.: Phys. Lett. A 335, 374 (2005)

[3] Kerimov, G. A.: J. Phys. A: Math. and Gen. 39, 1183 (2006)

[4] Khare, A., Bhaduri, R. K.: Am. J. Phys. 62, 1008 (1994)

[5] Kaushal, K. S.: Classical and Quantum Mechanics of Noncentral Potentials. SpringerVerlag, Berlin (1998)

[6] Yaşuk, F., Berkdemir, C., Berkdemir, A.: J. Phys. A: Math. and Gen. 38, 6579 (2005)

[7] Kandirmaz, N., Ünal, N.: Theor. and Math. Physics 155, 884 (2008)

[8] Aharonov, Y., Bohm D.: Phys. Rev. 115, 485 (1959)

[9] Dirac, P. A. M.: Proc. R. Soc. London Ser. A 133, 60 (1933)

[10] Makarov, A. A., Smorodinsky Y. A., Valiev K., Winternitz P.: Nuovo Cimento A 52, 1061 (1967)

[11] Quesne, C.: J. Phys. A: Math. and Gen. 21, 3093 (1988)

[12] Flügge, S.: Practical Quantum Mechanics I. Springer Verlag, Berlin (1971)

[13] Filho, H. B., Vaidya, A. N.: Phys. Lett. A 145, 69 (1990)

[14] Dong, S. H., Cassou, M. L.: Phys. Scripta 74, 285 (2006)

[15] Shahin, G. Y., Shikakhwa M. S.: J. Phys. A: Math. and Gen. 38, 759 (2005)

[16] Alhaidari, A. D., Bahlouli A., Hasan A.: Phys. Lett. A 349, 87 (2006)

[17] Dutra, A. S., Hott, M.: Phys. Lett. A 356, 215 (2006)

[18] Chen, C. Y.: Phys. Lett. A 339, 283 (2005)

[19] Chen, C. Y., Lu F. L., Sun D. S.: Phys. Lett. A 329, 420 (2004)

[20] Chen, C. Y., Sun, D. S., Liu C. L.: Phys. Lett. A 317, 80 (2003)

[21] Dong, C. Z., Gang, C.: Chin. Phys. Lett. 22, 795 (2005)

[22] Guo, J. Y., Han J. C., Wang R. D.: Phys. Lett. A 353, 378 (2006)

[23] Bordag, M., Voropaev, S.: J. Phys. A: Math. and Gen 26, 7637 (1993)

[24] Alhaidari, A. D.: Annals of Phys. 320, 453 (2005)

[25] Alhaidari, A. D.: Mod. Phys. Lett. A 21, 581 (2006) 
[26] Doebner, H. D., Papp, E.: Phys. Lett. A 144, 423 (1990)

[27] Alhaidari, A. D.: J. Phys. A: Math. and Gen. 38, 3409 (2005)

[28] Audretsch, J., Skarzhinsky, V. D., Voronov, B. L.: J. Phys. A: Math. and Gen. 34, 235 (2001)

[29] Gasaneo, G., Colavecchia, F. D., Cravero, W. R., Garibotti, C. R.: Phys. Rev. A 60, 284 (1999)

[30] Li, H., Wang, G., Ding, F., Wang J., Shen, W.: Phys. Lett. A 280, 325 (2001)

[31] Zhedanov, A. S.: J. Phys. A. Math. and Gen. 26, 4633 (1993)

[32] Kibler, M., Winternitz, P.: J. Phys. A: Math. and Gen. 20, 4097 (1987)

[33] Gerry, C. C.: Phys. Lett. A 118, 445 (1986)

[34] Dutt, R., Gangopadhyaya, A., Sukhatme, U. P.: Am. J. Phys. 65, 400 (1997)

[35] Gang, C.: Chin. Phys. 13, 144 (2004)

[36] Blado, G. G.: Inter. Jour. Quant. Chem. 58, 431 (1996)

[37] Gang, C.: Physica Scripta 70, 11 (2004)

[38] Cooper, F., Khare, A., Sukhatme, U.: Supersymmetry in Quantum Mechanics. World Scientific, Singapore ( 2001)

[39] Gönül, B., Zorba, İ.: Phys. Lett. A 269, 83 (2000)

[40] Gönül, B., Koçak, M.: Mod. Phys. Lett. A 20, 355 (2005)

[41] Koçak, M., Zorba, İ., Gönül, B.: Mod. Phys. Lett. A 17, 2127 (2002)

[42] Sökmen, I.: Phys. Lett. A 132, 65 (1988); 115, 249 (1986)

[43] Bornales, J., Bernido, C. C., Bernido, M. V. C.: Phys. Lett. A 260, 447 (1999)

[44] Basco, F., Bernido, C. C., Bernido, M. V. C.: Phys. Lett. A 157, 461 (1991)

[45] Bentag, B., Chetouni, L.: Czech. J. Phys. 50, 593 (2000)

[46] Cai, J. M., Inomata, A.: Phys. Lett. A 141, 315 (1989)

[47] C. Grosche, Phys. Lett. A 165 (1992) 185.

[48] Chetouni, L., Guechi, L., Hammann, T. F.: Phys. Lett. A 125, 277 (1987)

[49] Aktaş, M., Sever, R.: J. Math. Chem. 37, 139 (2005)

[50] Chen, C. Y., Liu, C. L., Sun, D. S.: Phys. Lett. A 305, 341 (2002) 
[51] Hartmann, H., Schuch, D.: Int. J. Q. Chem. XVIII, 125 (1980)

[52] Kibler, M., Negadi, T.: Int. J. Q. Chem. XXVI, 405 (1984)

[53] Kibler, M., Negadi, T.: Phys. Lett. A 124, 43 (1987)

[54] Souza, C. F., Gandelman, M., Albuquerque, L. C.: J. Phys. A: Math. and Gen. 22, L533 (1989)

[55] Bernido, M. V. C., Bernido, C. C.: Phys. Lett. A 134, 395 (1989)

[56] Granovskii, Y. I., Zhedanov, A. S., Lutzenko, I. M.: J. Phys. A: Math and Gen. 24, 3887 (1991)

[57] Guha A., Mukherjee, S.: J. Math. Phys. 28, 840 (1987)

[58] Yeşiltas Ö.: Chinese Phys. Lett. 25, 1172 (2008)

[59] Pantis G., Lagaris I. E., Sofinos S. A.: Phys. Rev. C 63, 044009 (2001)

[60] Pantis G., Fideldey H., Sprung D. W. L.: Z. Physik A 294, 101 (1980)

[61] Delfino A., Adhikari S. K., Tomio L., Frederico T.: Phys. Rev. C 46, 471 (1992)

[62] Nikiforov, A. F., Uvarov, V. B.: Special Functions of Mathematical Physics. Birkhäuser, Basel (1988) 